Cite this: Phys. Chem. Chem. Phys., 2014, 16, 9115

Received 20th January 2014, Accepted 21st March 2014

DOI: $10.1039 / c 4 c p 00294 f$

www.rsc.org/pccp

\section{Quantum dot-sensitized solar cells based on directly adsorbed zinc copper indium sulfide colloids $\uparrow$}

\begin{abstract}
Néstor Guijarro, $¥$ Elena Guillén,§ Teresa Lana-Villarreal and Roberto Gómez*
Heavy metal-based quantum dots (QDs) have been demonstrated to behave as efficient sensitizers in QDsensitized solar cells (QDSSCs), as attested by the countless studies and encouraging efficiencies reported so far. However, their intrinsic toxicity has arisen as a major issue for the prospects of commercialization. Here, we examine the potential of environmentally friendly zinc copper indium sulfide (ZCIS) QDs for the fabrication of liquid-junction QDSSCs by means of photoelectrochemical measurements. A straightforward approach to directly adsorb ZCIS QDs on $\mathrm{TiO}_{2}$ from a colloidal dispersion is presented. Incident photonto-current efficiency (IPCE) spectra of sensitized photoanodes show a marked dependence on adsorption time, with longer times leading to poorer performances. Cyclic voltammograms point to a blockage of the channels of the mesoporous $\mathrm{TiO}_{2}$ film by the agglomeration of QDs as the main reason for the decrease in efficiency. Photoanodes were also subjected to the ZnS treatment. Its effects on electron recombination with the electrolyte are analyzed through electrochemical impedance spectroscopy and photopotential measurements. The corresponding results bring out the role of the $\mathrm{ZnS}$ coating as a barrier layer in preventing electron leakage toward the electrolyte, as argued in other QD-sensitized systems. The beneficial effect of the ZnS coating is ultimately reflected in the power conversion efficiency of complete devices, reaching values of $2 \%$. In a more general vein, through these findings, we aim to call the attention to the potentiality of this quaternary alloy, virtually unexplored as a light harvester for sensitized devices.
\end{abstract}

\section{Introduction}

In the last few decades, concerns about the unavoidable collapse of the current fossil fuel economy have prompted the development of a wide variety of technologies aimed to convert solar energy into electricity in a cost-effective way. Among the third generation solar cells, currently under development, the so-called sensitized solar cells are drawing a lot of attention. ${ }^{1-3}$ The prospects of scalable and low cost fabrication via solution processing, together with their encouraging conversion efficiencies, make them promising candidates for feasibly exploiting solar energy.

Typically, the design of sensitized devices comprises a mesoporous wide band gap oxide film (electron acceptor) coated with

Institut Universitari d'Electroquimica i Departament de Quimica Fisica, Universitat d'Alacant, Apartat 99, E-03080 Alacant, Spain.E-mail: nestor.guijarro@epfl.ch, roberto.gomez@ua.es; Fax: +3496590 3537; Tel: +34 965903748

$\dagger$ Electronic supplementary information (ESI) available: Experimental details of the synthesis of ZCIS colloidal QDs and estimate of ZCIS energy levels. See DOI: 10.1039/c4cp00294f

\# Present address: Laboratory for Molecular Engineering of Optoelectronic Nanomaterials, Institute of Sciences and Engineering, École Polytechnique Fédérale de Lausanne, Station 6, CH-1015 Lausanne, Switzerland.

$\S$ Present address: Abengoa Research, C/Energía Solar 1, Campus Palmas Altas, 41014, Sevilla, Spain. a light harvester material (sensitizer), in contact with a hole acceptor phase (viz. a redox couple in a liquid electrolyte, or a solid hole conductor). ${ }^{2,4}$ Under operation, the exciton generated in the sensitizer upon photon absorption is split by the injection of an electron and a hole into the oxide and the hole scavenger, respectively, followed by carrier transport toward opposite collectors. Obviously, the relative band positions and the electrical coupling among different components have proven to critically govern the charge separation and transport dynamics, and hence, the overall conversion efficiency. ${ }^{5}$ Up to now, mostly dye molecules and semiconductor QDs have been tested as sensitizers. It must be noted that very recently hybrid inorganic-organic perovskites have also been coupled to nanostructured oxides yielding outstanding performances, though their role as sensitizers is still under discussion. ${ }^{6,7}$ Dye-sensitized solar cells have delivered impressive efficiencies of up to $12.3 \%,{ }^{8}$ whereas the performance of QD-sensitized solar cells lags far behind these values.,10 Nonetheless, the stunning properties of QDs, such as the tunable band gap, high extinction coefficient, and the unparalleled prospect of multiple exciton generation (MEG) still leave room for improvement. ${ }^{11,12}$

Recent findings compellingly suggest that a tight control of the surface properties of the QDs, together with a better 
understanding of the interfacial charge transfer dynamics, holds the key to further improve the conversion efficiency of QDSSCs. ${ }^{9,13-18}$ In this sense, it has lately been recognized that the use of presynthesized colloidal QDs, instead of in situ deposited ones, is preferable to perform sensitization. ${ }^{19,20}$ In situ deposition methods, such as chemical bath deposition (CBD) and successive ionic layer adsorption and reaction (SILAR), allow us to efficiently coat nanoporous structures, but the lack of control over the nanocrystal size and composition, besides the high degree of aggregation, hampers charge separation while enhancing dramatically recombination. ${ }^{17,19,21,22}$ By contrast, highly crystalline surface-passivated colloidal QDs have shown greater yields of charge injection, especially when attached in a linkerless fashion, as otherwise the linkers tethering the QDs to the oxide could work as recombination centers. ${ }^{23,24}$ Note that, to date, MEG has only been reported on systems sensitized with colloidal QDs, primarily because of the lower density of defects at the heterojunction. ${ }^{25,26}$ Unfortunately, the unsatisfactorily low QD coverage is still a major challenge limiting the conversion of these devices. ${ }^{24}$

Interestingly, several photoanode post-treatments have arisen as powerful tools to improve the photovoltaic characteristics by precisely tuning the interfacial properties. First, the adsorption of dipole molecules on QDs has been reported to modify the band alignment and to mediate hole extraction. ${ }^{27-29}$ Second, short annealing treatments have improved electron coupling at the $\mathrm{TiO}_{2} / \mathrm{QD}$ interface. ${ }^{13}$ And third, the ubiquitous $\mathrm{ZnS}$ coating has demonstrated to drastically reduce electron leakage to the electrolyte. ${ }^{14}$ In any case, there are still some open questions about the basic functioning of QDSSCs, such as the role of QD surface states in charge separation and recombination processes. ${ }^{30,31}$

The conversion efficiency of liquid junction QDSSCs has experienced an impressive increase in the last few years, also when using colloidal QDs. The record efficiency of $1.83 \%$ set by Giménez et al. in $2009^{24}$ has recently been beaten by Wang et al., achieving values above $6 \% .{ }^{10}$ However, the intrinsic toxicity of commonly used heavy metal-based chalcogenide QDs (viz. CdS, CdSe, $\mathrm{CdSe}_{x} \mathrm{Te}_{1-x}$, $\mathrm{PbS}, \mathrm{PbSe}$ ) has come out as a major obstacle for an eventually large-scale commercial expansion of these devices. In this respect, and well before the concept of QDSSCs burst in the field of photovoltaics, $\mathrm{CuInS}_{2}$ (CIS) emerged as a promising environmentally benign photoactive material. ${ }^{32}$ Indeed, its inherent characteristics such as a large extinction coefficient $\left(\sim 5 \times 10^{5} \mathrm{~cm}^{-1}\right.$, one order of magnitude higher than that of bulk CdSe $)^{33}$ or a direct band gap of $1.52 \mathrm{eV}$ (in the optimum range for harvesting most of the solar spectrum) account for the countless studies on this material. ${ }^{34}$ Thin film solar cells based on CIS absorber layers have been reported since the 1970 s, yielding record efficiencies of up to $12.5 \% .{ }^{35}$ Unfortunately, the necessity of circumventing the segregation of $\mathrm{Cu}_{x} \mathrm{~S}$ and $\mathrm{In}_{2} \mathrm{~S}_{3}$ phases during the preparation of the films, which critically deteriorates device performance, increases fabrication costs, hampering, in turn, the commercialization of CIS-based devices. ${ }^{36}$

Recent interest in nanometer-sized materials has boosted the development of novel low-cost synthetic routes for the preparation of CIS QDs, free from phase segregation issues, as well as methodologies for their implementation in QDSSCs. Li et al. sensitized $\mathrm{TiO}_{2}$ with CIS colloidal QDs prepared by a solvothermal method, using mercaptopropionic acid (MPA) as a linker, and reported conversion efficiencies of $4.2 \% .^{37,38}$ More recently, Santra et al. electrophoretically deposited CIS QDs on the $\mathrm{TiO}_{2}$ surface, delivering efficiencies close to $4 \% .{ }^{39}$ Likewise, Luo et al. prepared aqueous colloidal dispersions of mercaptoacetic acid-capped CIS QDs that, subsequently, were employed to modify the $\mathrm{TiO}_{2}$ surface, yielding efficiencies above $5 \% .{ }^{40}$ Aside from these reports dealing with colloidal CIS QDs, several authors have managed to directly grow this ternary material by SILAR methods in the matrix oxide. ${ }^{41,42}$ Particularly interesting is the work of Chang et al., where a $2.5 \%$ solar-power conversion efficiency was claimed. ${ }^{41}$ However, all these values should be taken with caution, since in all cases additional layers of CdS, or $\mathrm{Cu}_{2} \mathrm{~S}$ and $\mathrm{ZnSe}$, which can evidently work as co-sensitizers (not only as buffer layers to promote charge extraction or surface passivation), are required to attain efficiencies beyond one percent. The poor performance of bare CIS-sensitized devices is believed to be caused by an unmatched band alignment across the $\mathrm{TiO}_{2} / \mathrm{CIS}$ interface. ${ }^{43}$ Obviously, size confinement allows for widening the bandgap, enhancing thereby the driving force for injection, but at the cost of wasting most of the photons of the solar spectrum. Interestingly, alloying CIS QDs with ZnS (bandgap of $3.60 \mathrm{eV}$ ) has turned out to be a useful strategy to both passivate surface trap states and, which is most alluring, to tune the energy level positions. ${ }^{44,45}$ Interestingly, Kuo et al. evidenced that the quaternary alloy based on ZnS/CIS behaves as a sensitizer, delivering conversion efficiencies of $0.7 \%$ when $\mathrm{ZnS} / \mathrm{CIS}$ (ZCIS) QDs were tethered via mercaptopropionic acid to $\mathrm{ZnO}$ nanowires in the presence of an iodide/iodine-based redox electrolyte. ${ }^{46}$ To the best of our knowledge, apart from this publication no more attempts addressing sensitization with ZCIS QDs have been reported to date.

Pursuing to further exploit this quaternary QD in sensitized devices, we report on a straightforward procedure for preparing ZCIS-sensitized $\mathrm{TiO}_{2}$ photoanodes, whereby colloidal QDs are assembled on the oxide in a linkerless fashion. The performance of these photoanodes is examined in a liquid junction configuration, by using typical redox electrolytes for QDSSCs (sulfide and polysulfide). Incident-photon to current-efficiency (IPCE) measurements are carried out to unveil the effect of the QD coverage on the charge separation yield. The effect of a $\mathrm{ZnS}$ coating on the performance of QDSSCs is analyzed by means of electrochemical impedance spectroscopy (EIS) and photopotential measurements. In a more general vein, the potentiality of ZCIS QDs as a feasible environmentally friendly alternative to heavy metal-based QDs in sensitized devices is discussed in terms of solar-to-power conversion.

\section{Experimental}

\section{Synthesis of zinc copper indium sulfide colloidal QDs}

Zinc copper indium sulfide (ZCIS) QD colloidal dispersions were prepared by following the procedure described by Nakamura et al. ${ }^{47}$ 
with minor changes, as recently reported by some of us. ${ }^{48}$ The as-synthesized QDs were washed by precipitating them with a mixture of $1: 4 \mathrm{CH}_{2} \mathrm{Cl}_{2}$ : EtOH followed by centrifugation, and then they were redispersed in $\mathrm{CH}_{2} \mathrm{Cl}_{2}$. Note that this process was carried out just once, as repetition of the washing procedure gives rise to insoluble particles, likely due to irreversible loss of the capping agent and subsequent aggregation. Further characterization of the QDs can be found in ref. 48 .

\section{Preparation of $\mathrm{TiO}_{2}$ films}

Nanoporous $\mathrm{TiO}_{2}$ electrodes were prepared by spreading an aqueous slurry of Degussa P25 nanoparticles over either an $\mathrm{F}: \mathrm{SnO}_{2}$ (FTO) coated glass or a thermally treated titanium foil. ${ }^{16}$ Briefly, $1 \mathrm{~g}$ of $\mathrm{TiO}_{2}$ powder was mixed and ground with $1.5 \mathrm{~mL}$ of $\mathrm{H}_{2} \mathrm{O}, 30 \mu \mathrm{L}$ of acetylacetone, and $30 \mu \mathrm{L}$ of Triton X100. Typically, $8 \mu \mathrm{L} \mathrm{cm}{ }^{-2}$ were applied by doctor blading on each substrate, followed by sintering for $1 \mathrm{~h}$ at $450{ }^{\circ} \mathrm{C}$ in air. The $\mathrm{TiO}_{2}$ film thickness was determined to be $\sim 11 \mu \mathrm{m}$ using a profilometer KLA Tencor D-100.

\section{ZCIS assembly on $\mathrm{TiO}_{2}$ films and glass}

Sensitization of $\mathrm{TiO}_{2}$ samples by direct adsorption of ZCIS QDS was carried out by immersing the oxide films in a newly washed $\mathrm{CH}_{2} \mathrm{Cl}_{2}$ colloidal dispersion, using soaking times ranging from a few minutes to overnight. Glass/ZCIS samples were prepared by (i) drop casting the dichloromethane colloidal dispersion on glass, followed by (ii) drying in air and (iii) immersion in ethanol for $15 \mathrm{~s}$ to remove the capping ligand.

\section{Surface post-treatment: ZnS treatment}

The ZnS coating was performed by following the optimized SILAR method proposed by Diguna et al. ${ }^{49}$ This procedure, repeated twice, entails consecutively dipping sensitized samples in $0.5 \mathrm{M} \mathrm{Zn}\left(\mathrm{CH}_{3} \mathrm{COO}\right)_{2}$ and in $0.5 \mathrm{M} \mathrm{Na}_{2} \mathrm{~S}$ aqueous solutions for $1 \mathrm{~min}$, washing thoroughly with water between immersions to remove the excess of non-adsorbed/unreacted ions.

\section{Optical characterization: absorption spectra}

Steady-state absorption and emission spectra were obtained for both colloidal dispersions and sensitized films. UV-Vis absorption spectra were recorded using a Shimadzu UV-2401PC spectrophotometer. Specifically, diffuse reflectance spectra were measured for scattering sensitized $\mathrm{TiO}_{2}$ electrodes using an integrating sphere with $\mathrm{BaSO}_{4}$ as a background. The KubelkaMunk transformation was undertaken to facilitate the interpretation of reflectance data.

\section{Photoelectrochemical characterization of photoanodes}

Photoelectrochemical measurements were performed at room temperature using a three-electrode cell with a fused silica window and a computer-controlled Autolab PGSTAT30 potentiostat. In all the cases, an $\mathrm{Ag} / \mathrm{AgCl} / \mathrm{KCl}$ (sat) electrode and a Pt wire were employed as a reference and counter electrode, respectively. All the potentials are referred to the $\mathrm{Ag} / \mathrm{AgCl} / \mathrm{KCl}$ (sat) electrode. Two different working electrolytes were used: a $0.5 \mathrm{M} \mathrm{Na}_{2} \mathrm{~S}+1 \mathrm{M}$ $\mathrm{NaOH}$ solution, and a $1 \mathrm{M} \mathrm{Na}_{2} \mathrm{~S}+0.1 \mathrm{M} \mathrm{S}+1 \mathrm{M} \mathrm{NaOH}$ solution (polysulfide), both prepared with ultrapure water (Millipore Elix 3) and $\mathrm{N}_{2}$-purged before measurements. Cyclic voltammetry was carried out in the dark at a scan rate of $20 \mathrm{mV} \mathrm{s}^{-1}$. Electrochemical Impedance Spectroscopy (EIS) measurements were also performed in the dark, using a frequency range from $10 \mathrm{mHz}$ to $10 \mathrm{kHz}$, at $-1.2 \mathrm{~V}$ with a perturbation of $10 \mathrm{mV}$. A $300 \mathrm{~W}$ Xe arc lamp (Thermo Oriel) was employed for electrode illumination. Incident-photon to current-efficiency (IPCE) measurements were carried out by placing a monochromator (Oriel model 74100) between the white light source and the cell, illuminating the electrode from the substrate side. The light intensity was measured by coupling an optical power meter (Oriel model 70310) with a thermopile (Thermo Oriel 71964). In the case of photopotential measurements, the full output of the lamp equipped with a UV-filter (cut-off $\lambda<380 \mathrm{~nm}$ ), a water filter (to remove IR radiation), and a neutral density filter $(\mathrm{OD}=0.5)$ was employed for electrode illumination, yielding an irradiance of $48 \mathrm{~mW} \mathrm{~cm} \mathrm{~cm}^{-2}$ measured using an optical power meter equipped with a thermopile.

\section{Fabrication and characterization of complete devices}

Liquid electrolyte based solar cells were prepared by sandwiching a ZCIS-sensitized $\mathrm{TiO}_{2}$ photoanode and a copper sulfide cathode, using a silicone spacer (thickness $\sim 50 \mu \mathrm{m}$ ), and a droplet $(10 \mu \mathrm{L})$ of polysulfide. The active area was $0.24 \mathrm{~cm}^{2}$ in all the cases. EIS measurements were carried out in the dark, under different forward bias with a frequency range of $10 \mathrm{mHz}$ to $10 \mathrm{kHz}$, and an $\mathrm{AC}$ amplitude of $10 \mathrm{mV}$. Impedance spectra were fitted to the model proposed by González-Pedro et al. ${ }^{50}$ The photovoltaic characteristics were obtained by illuminating the complete devices using a Sun 2000 solar simulator (ABET technologies) at 1 sun (AM $1.5 \mathrm{G}, 100 \mathrm{~mW} \mathrm{~cm}^{-2}$ ).

\section{Results and discussion}

Fig. 1A shows the absorption spectrum of the ZCIS colloidal dispersion employed in this study, characterized by an absorption onset at around $650 \mathrm{~nm}$, and a broad, but distinct, band at $545 \mathrm{~nm}$, which suggests a rather narrow size distribution. The absorption spectra of ZCIS-sensitized films as a function of the adsorption time in Kubelka-Munk units are depicted in Fig. 1B. As expected, the longer the adsorption time, the higher the QD loading, which results in an increasingly reddish coloration until saturation (overnight immersion). It is apparent that the spectra of sensitized $\mathrm{TiO}_{2}$ films inherit the absorption characteristics of colloidal QDs, in agreement with other studies reported elsewhere. ${ }^{23,24}$ Note that CdSe-sensitized $\mathrm{TiO}_{2}$ films have already been prepared by a similar linkerless approach. ${ }^{23}$ Interestingly, this points out that, regardless of the nature of the QDs, direct anchorage can be performed by optimizing washing procedures, and carefully selecting the solvent for dispersing the QDs.

In order to shed some light on the morphology of the nanocrystals, transmission electron microscopy (TEM) images of the colloidal dispersion and sensitized films were recorded. 


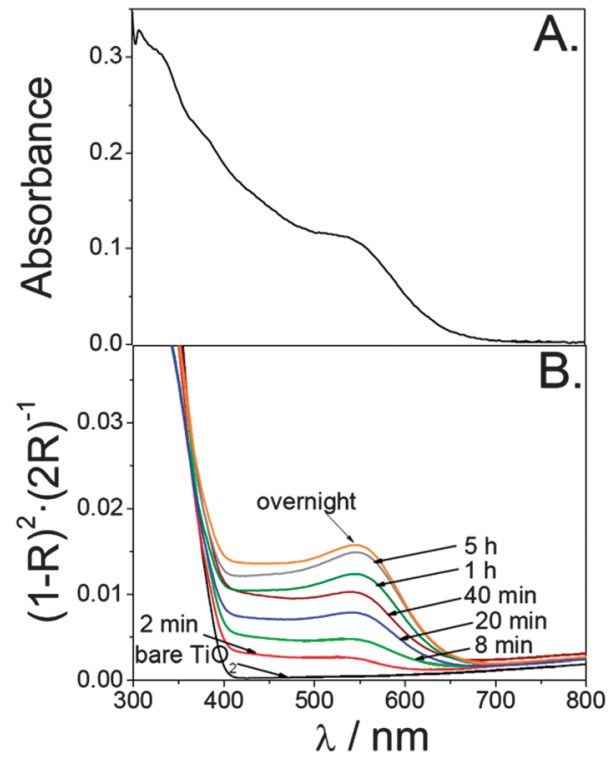

Fig. 1 Absorption spectrum of a ZCIS QD colloidal dispersion in $\mathrm{CH}_{2} \mathrm{Cl}_{2}$ (A) and Kubelka-Munk transformation of the diffuse reflectance spectra obtained for $\mathrm{TiO}_{2}$ nanoporous thin films sensitized with ZCIS QDs using different adsorption times (B).

As noticed in Fig. 2A, dispersed ZCIS QDs appear to be spherical, with an average diameter of $4 \mathrm{~nm}$. Upon sensitization, $\mathrm{TiO}_{2}$ nanoparticles become decorated by ZCIS QDs, although a low coverage seems to be attained, even at saturation (Fig. 2B). However, it is worth noting that a small portion of the sample was detached

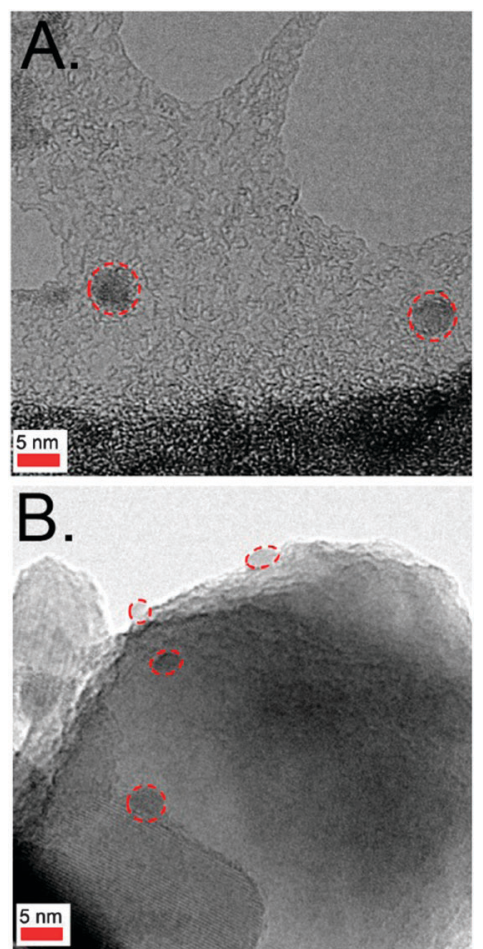

Fig. 2 TEM images of ZCIS colloids (A) and ZCIS-sensitized $\mathrm{TiO}_{2}$ (B) (overnight adsorption) samples. Dashed lines are used as a guide for the eye, indicating the location of the QDs. from the conducting glass substrate to acquire the TEM images. This could have entailed a distortion of the original structure of the sample. In fact, electrochemical experiments seem to indicate that the actual coverage is much higher in the uppermost part of the sensitized film (see below). In any case, it has been already reported that QD adsorption from organic solvents gives rise to maximum QD coverages ${ }^{23}$ lower than those obtained from aqueous medium. ${ }^{13}$ Admittedly, TEM images reported by Kuo et al. for sensitized samples clearly reveal that linker-mediated adsorption of ZCIS QDs from aqueous medium allows for a much higher QD loading. ${ }^{46}$ However, direct anchorage is obviously much simpler, avoiding ligand exchange treatments and, more importantly, it permits an intimate contact between the oxide and the QDs, which is crucial for efficient charge separation. ${ }^{16}$

The ability of ZCIS QDs to behave as sensitizers was evaluated by measuring the IPCE of ZCIS-modified electrodes, using an aqueous sulfide electrolyte as a hole scavenger (Fig. 3A). At a first sight, it is evident that the IPCE spectra preserve the features of the absorption spectra of the sensitized electrodes and that their values reflect a marked dependence on adsorption time. For the sake of clarity, the evolution of IPCE values measured at $550 \mathrm{~nm}$ has been plotted against absorption (in Kubelka-Munk units) in Fig. 3A (inset). As observed, the IPCE increases with the amount of QDs, reaching a maximum value of $15 \%$ after $1 \mathrm{~h}$ adsorption and then decreasing for longer sensitization times. It must be underlined that, in spite of the low QD coverage, relatively high IPCE values
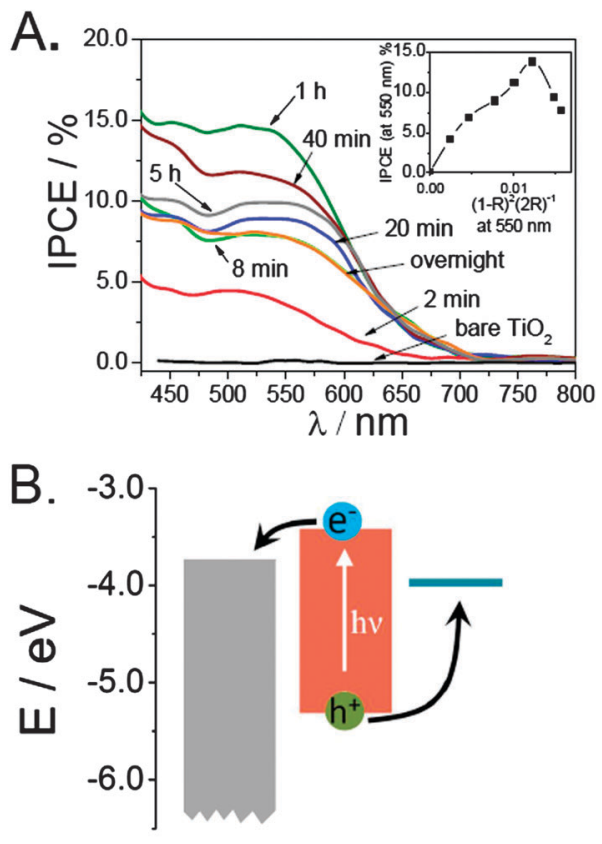

\section{$\mathrm{TiO}_{2} \quad \mathrm{ZCIS} \quad \mathrm{Na}_{2} \mathrm{~S}$}

Fig. 3 IPCE spectra of $\mathrm{TiO}_{2}$ electrodes sensitized through direct adsorption of ZCIS QDs for different adsorption times (labeled), all measurements were performed in an $\mathrm{N}_{2}$-purged $0.5 \mathrm{M} \mathrm{Na}_{2} \mathrm{~S}+1 \mathrm{M} \mathrm{NaOH}$ aqueous electrolyte (A), and IPCE vs. electrode absorption (in Kubelka-Munk units) at $550 \mathrm{~nm}$ (inset). Energy level diagram estimated for the $\mathrm{TiO}_{2} / \mathrm{ZCIS}$ assembly in a $\mathrm{Na}_{2} \mathrm{~S}$ electrolyte (B). 
are attained, suggesting that charge separation across both $\mathrm{TiO}_{2} / \mathrm{ZCIS}$ and ZCIS/sulfide interfaces occurs in an efficient way. This is in good agreement with the energy diagram depicted in Fig. 3B, where a rather large driving force of $320 \mathrm{meV}$ for electron injection, and above $1 \mathrm{eV}$ for hole withdrawal are estimated (see ESI $\dagger$ ).

Interestingly, the trend in the IPCE spectra as a function of the adsorption time resembles that previously reported for the direct adsorption of colloidal CdSe QDs. ${ }^{23}$ The progressive increase in the IPCE with the adsorption time is not unexpected, since a higher amount of sensitizer would promote an enhanced light harvesting. In the case of CdSe QDs, the deterioration of the conversion efficiency for long adsorption times was attributed to the aggregation of QDs. More specifically, QD aggregation could decrease the electron injection yield in two different manners. On the one hand, QD/QD interfaces can actively participate in carrier trapping and recombination, slowing down electron injection from either QDs in direct contact with the oxide, or from those localized in outer layers. ${ }^{16}$ On the other hand, massive agglomeration at the entrance of the nanochannels of the nanoporous oxide scaffold can hinder the entry of the hole scavenger, rendering inactive a significant part of the electrode. Given that injection across QD multilayers indeed occurs to some extent, ${ }^{30,51}$ we speculate that the deterioration of the photonto-current conversion could be mainly attributed to the blockage of the nanochannels.

To elucidate how the QD loading affects the electrode active surface area, cyclic voltammograms of the sensitized electrodes were recorded in an aqueous sulfide electrolyte for different adsorption times. As shown in Fig. 4A, cyclic voltammograms are almost reversible in all cases, although the capacitance in the accumulation region becomes smaller as the amount of sensitizer increases. Keeping in mind that the capacitive currents are proportional to the electrochemically active interfacial area of the electrode, ${ }^{52,53}$ the progressive decrease of such currents clearly indicates a partial loss of active surface area for long QD adsorption times. This finding supports that ZCIS QD agglomeration on top of the $\mathrm{TiO}_{2}$ film is the main cause for the efficiency deterioration. Alternatively, one could hypothesize that an upward shift of the $\mathrm{TiO}_{2}$ conduction band edge toward more negative potentials might be induced by equilibration processes between $\mathrm{TiO}_{2}$ and ZCIS QDs after coupling, bringing about an apparent shrinking of the capacitive region. As shown in Fig. 4B, multiplication of the negative-going voltammetric curve obtained for the sample sensitized overnight by 1.6 results in a curve that virtually overlaps that obtained for bare $\mathrm{TiO}_{2}$. These results provide compelling evidence that the location of the conduction band remains unaltered, whereas the drop in the $\mathrm{TiO}_{2}$ density of states observed by cyclic voltammetry is connected to the decrease of the $\mathrm{TiO}_{2}$ volume contributing to the capacitance. It is worthwhile underlining that both the IPCE and cyclic voltammetry dependencies on the QD loading are remarkably similar to those reported for the direct adsorption of CdSe QDs on $\mathrm{TiO}_{2}{ }^{23}$ This suggests that the mechanism of ZCIS QD adsorption consists in the initial formation of a sub-monolayer,

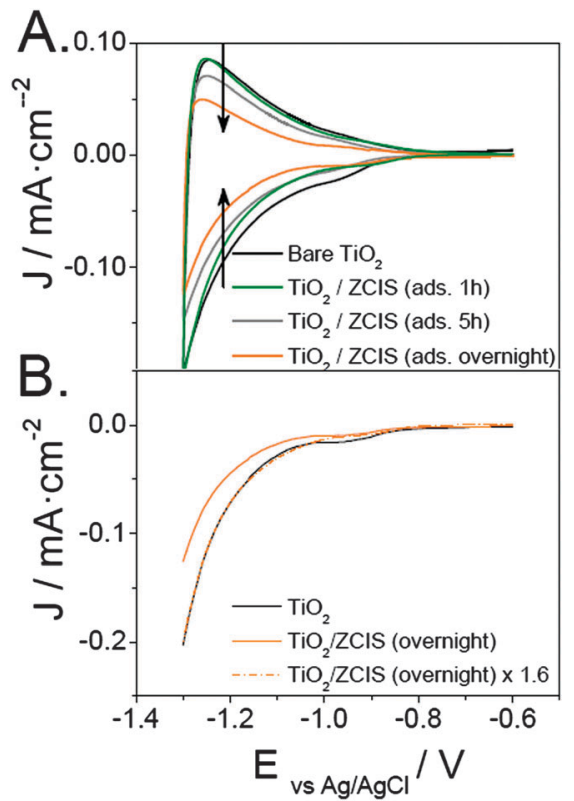

Fig. 4 Cyclic voltammograms of $\mathrm{TiO}_{2}$ electrodes modified by direct adsorption of ZCIS QDs as a function of the adsorption time (A). Comparison between the negative-going scan for bare and ZCIS-sensitized (overnight) $\mathrm{TiO}_{2}$ electrodes (B). All cyclic voltammograms were recorded at $20 \mathrm{mV} \mathrm{s}^{-1}$ in the dark in an $\mathrm{N}_{2}$-purged $0.5 \mathrm{M} \mathrm{Na}_{2} \mathrm{~S}+1 \mathrm{M} \mathrm{NaOH}$ aqueous solution.

followed by aggregation, as recently argued by Pernik et al. for CdSe sensitization. ${ }^{54}$ Given that the adsorption time of $1 \mathrm{~h}$ yields the best IPCE values, the electrodes examined henceforth were prepared in this way.

We next consider the effects of the photoanode surface treatment with ZnS on the optical and interfacial charge transfer properties of the ZCIS-modified samples. Fig. 5 shows that the ZnS coating induces a slight red shift $(\sim 8 \mathrm{~nm})$ and a broadening of the band centered at around $545 \mathrm{~nm}$, irrespective of the substrate employed (flat glass or mesoporous $\mathrm{TiO}_{2}$ ). It has been argued that $\mathrm{ZnS}$ covers not only the bare $\mathrm{TiO}_{2}$ (or glass) substrate, but also the QDs. ${ }^{19}$ Consequently, the ZnS shell grown over the QDs allows us to extend the electronic wave function within a larger distance, which accounts for a partial loss of quantum confinement. ${ }^{14}$

On the other hand, to gain some insight into the effect of the ZnS coating on the photoanode performance, open circuit potential (OCP) measurements under transient illumination and electrochemical impedance spectroscopy were performed in polysulfide in a three-electrode cell configuration. As shown in Fig. 6A, upon $\mathrm{ZnS}$ deposition both the more negative steadystate OCP under illumination, and the slower relaxation when the light is interrupted are recorded. The former reflects that a higher electron concentration can be accumulated in the $\mathrm{TiO}_{2}$ film, whereas the latter evidences a slowdown in the kinetics of electron recombination with the electrolyte. These results support the notion that $\mathrm{ZnS}$ acts as a barrier layer preventing electron leakage to the electrolyte. Likewise, impedance spectra in the dark for both bare and ZCIS-sensitized $\mathrm{TiO}_{2}$ films, with 


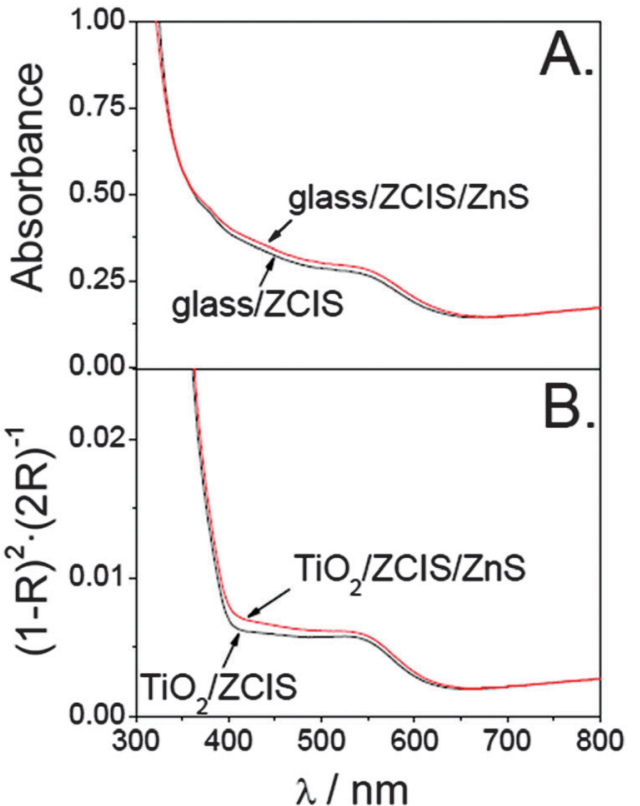

Fig. 5 Absorbance for ZCIS QDs deposited on glass before and after the ZnS treatment (A). Kubelka-Munk transformation of the diffuse reflectance spectra of a $\mathrm{TiO}_{2}$ film sensitized with ZCIS QDs, before and after ZnS deposition (B).

and without the $\mathrm{ZnS}$ treatment, are depicted in Fig. 6B. In all cases, one semi-arc is observed in the Nyquist plot in the middle-frequency region, which is attributed to electron transfer across the $\mathrm{TiO}_{2} / \mathrm{QD} /$ electrolyte interface. Noticeably, the semi-arc radius increases when the bare $\mathrm{TiO}_{2}$ surface is sequentially modified with ZCIS and ZnS. Qualitatively, the larger the semi-arc radius, the higher the charge transfer resistance. On this basis, not only the ZnS layer, but also the ZCIS QDs, acts as a barrier hampering the transfer of the electrons accumulated in the film to oxidized species in the electrolyte, the effectiveness of ZnS being clearly higher. Interestingly, similar results have recently been reported for CdSe-sensitized photoanodes,${ }^{14,55}$ pointing to the universality of the ZnS treatment for reducing electron recombination with the electrolyte.

Sandwich-type solar cells were fabricated by clipping a photoanode and a $\mathrm{Cu}_{2} \mathrm{~S}$ cathode, using polysulfide as a hole transporting medium. EIS measurements were performed in the dark as a function of the applied bias, fitting the experimental data to the model proposed by González-Pedro et al. ${ }^{50}$ to extract the charge transfer resistance (Fig. 7A). In agreement with the results described above for the three-electrode cell configuration, charge transfer resistance is more than doubled upon the ZnS treatment for the different applied bias. An increase in the forward bias triggers an upward shift of the photoanode Fermi level, which accounts for the progressive decrease in charge transfer resistance as reported elsewhere. ${ }^{55}$ The $J-V$ curves corresponding to solar cells before and after ZnS modification are presented in Fig. 7B. At a glance, the performance of the ZCIS-sensitized device is clearly enhanced upon the ZnS coating. More specifically, the photovoltaic parameters of the ZnS-treated cell $\left(J_{\mathrm{sc}}=6.83 \mathrm{~mA} \mathrm{~cm}{ }^{-2}, V_{\mathrm{oc}}=0.56\right.$,
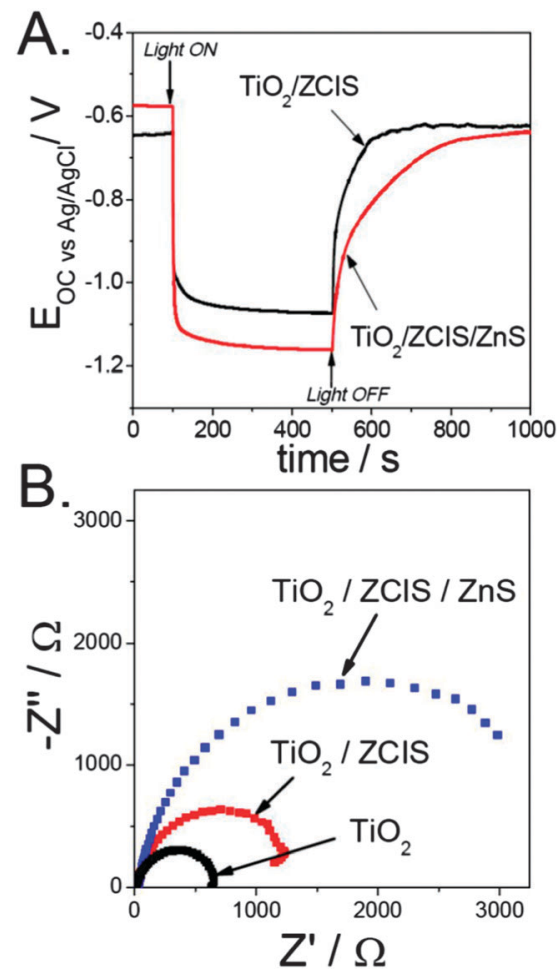

Fig. 6 Open circuit potential measurements obtained for transient illumination of ZCIS-sensitized electrodes (A). Irradiance: $48 \mathrm{~mW} \mathrm{~cm}$. Electrochemical impedance spectra of $\mathrm{TiO}_{2}$ electrodes sequentially modified with ZCIS QDs and ZnS (B). The data were obtained in the dark, in the range of $10 \mathrm{mHz}$ to $10 \mathrm{kHz}$ at $-1.2 \mathrm{~V}$, and with an oscillation amplitude of $10 \mathrm{mV}$. All the electrodes were prepared using thermally treated titanium foil as a substrate, and measured in an $\mathrm{N}_{2}$-purged $1 \mathrm{M} \mathrm{Na}_{2} \mathrm{~S}+0.1 \mathrm{MS}+1 \mathrm{M}$ $\mathrm{NaOH}$ aqueous electrolyte.

$\mathrm{FF}=0.56$ and $\eta=2.01 \%)$ under simulated AM 1.5 G illumination $\left(100 \mathrm{~mW} \mathrm{~cm}^{-2}\right)$ outperform those obtained for the initial device: $J_{\mathrm{sc}}=4.34 \mathrm{~mA} \mathrm{~cm}{ }^{-2}, V_{\mathrm{oc}}=0.51, \mathrm{FF}=0.57$ and $\eta=1.26 \%$. Essentially, the minimization of the electron leakage toward the electrolyte is commonly believed to be mainly responsible for the enhancement of the short circuit current and the open circuit voltage, giving rise to a higher conversion efficiency. ${ }^{55}$ It is worth comparing these results with those previously reported by Kuo et al. for ZCIS-sensitized devices. ${ }^{46}$ These authors attained efficiencies of $0.71 \%$, rather low, taking into account the high QD loading inferred from their TEM images and photoanode pictures. The different mode of attachment (direct vs. linker-assisted adsorption) and electrolyte (polysulfide vs. iodine/iodide solution) could explain the disparity. On the one hand, the direct adsorption of colloidal QDs has proven to lead to higher yields of charge separation than linker-mediated adsorption. Basically, the attachment in a linkerless fashion allows for an intimate contact between the QDs and the $\mathrm{TiO}_{2}$, whereas the introduction of a linker in between increases the electron donor-acceptor distance, decreasing the rate of injection. ${ }^{16}$ Moreover, the role of the linker molecules as recombination centers that decreases the overall charge separation has previously been disclosed. ${ }^{16}$ On the other hand, iodine/iodidebased electrolytes are known to corrode chalcogenide QDs 

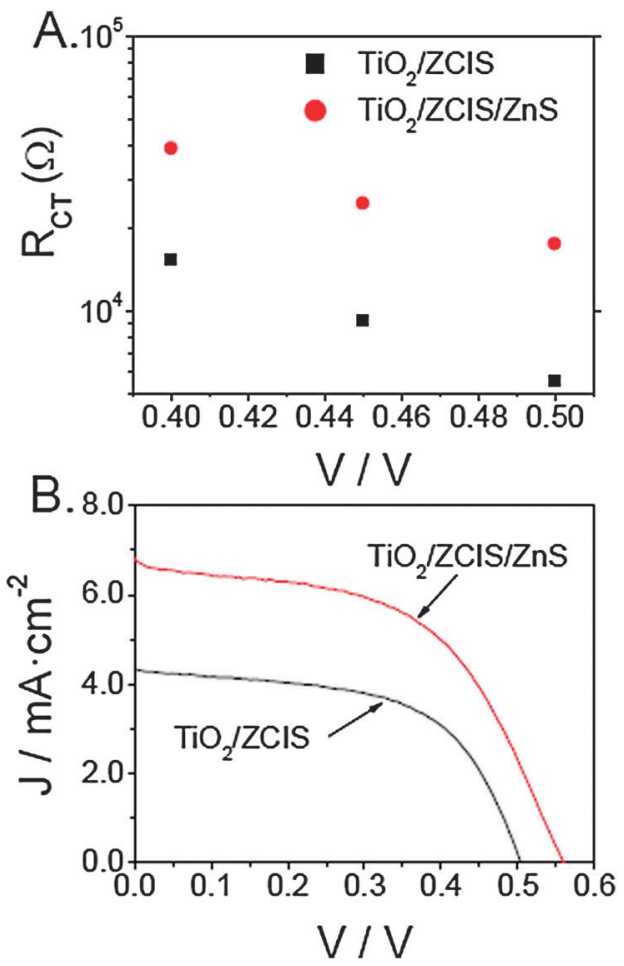

Fig. 7 Charge transfer resistance $\left(R_{C T}\right)$ as a function of the applied bias in a sandwich-type solar cell obtained by fitting the experimental EIS data to the model proposed by González-Pedro et al. ${ }^{50}$ (A). J-V curves of QDSSCs using either $\mathrm{TiO}_{2} / \mathrm{ZCIS}$ or $\mathrm{TiO}_{2} / \mathrm{ZCIS} / \mathrm{ZnS}$ as photoanodes (B).

unlike polysulfide.$^{56}$ It must be noted that a comparison with CIS-sensitized devices points to the fact that the ZCIS quaternary compound gives rise to better conversion efficiencies in the absence of buffer layers. The increase in performance observed when alloying CIS with $\mathrm{ZnS}$ matches with the predicted upward shift of the conduction band of the QDs, ${ }^{47,57}$ causing larger driving force for electron injection.

\section{Conclusions}

A straightforward procedure for the direct adsorption of ZCIS QDs at mesoporous $\mathrm{TiO}_{2}$ films is described, offering a convenient alternative to the linker-mediated attachment reported for this sensitizer so far. IPCE spectra of ZCIS-sensitized films display a strong dependence on the QD adsorption time, decreasing the overall conversion efficiency for longer adsorption times. Cyclic voltammograms suggest that the blockage of the nanochannels of the mesoporous $\mathrm{TiO}_{2}$ scaffold caused by QD aggregation is behind the performance detriment. The presence of a $\mathrm{ZnS}$ coating significantly improves the power conversion efficiency, reaching values around $2 \%$. Both impedance and photopotential measurements complementarily bring out that the ZnS layer covering the photoanode surface functions as a blocking layer, increasing the resistance of electron recombination with the electrolyte. The present findings demonstrate the potential of ZCIS QDs as a promising "green" alternative to heavy metal-based semiconductor sensitizers, and even to its ternary counterpart CIS. Further work is in progress to increase the QD coverage in the nanoporous scaffold as a way to further enhance the device efficiency. In a more general vein, the overall correspondence between the results reported in this work and those obtained for colloidal CdSe-sensitized devices clearly reflects generalities in the importance of obtaining both an accurate control of interfaces in QDSSCs and a high level of understanding of these devices.

\section{Acknowledgements}

N.G. is grateful to the Spanish Ministry of Education for the award of an FPU grant. The group acknowledges support of the Spanish Ministry of Economy and Competitiveness through projects HOPE CSD2007-00007 (Consolider Ingenio 2010) and MAT2012-37676 (Fondos FEDER).

\section{Notes and references}

1 B. E. Hardin, H. J. Snaith and M. D. McGehee, Nat. Photonics, 2012, 6, 162-169.

2 S. Rühle, M. Shalom and A. Zaban, ChemPhysChem, 2010, 11, 2290-2304.

3 J. Burschka, N. Pellet, S.-J. Moon, R. Humphry-Baker, P. Gao, M. K. Nazeeruddin and M. Grätzel, Nature, 2013, 499, 316-319.

4 B. O'Regan and M. Grätzel, Nature, 1991, 353, 737-740.

5 I. Robel, M. Kuno and P. V. Kamat, J. Am. Chem. Soc., 2007, 129, 4136-4137.

6 M. M. Lee, J. Teuscher, T. Miyasaka, T. N. Murakami and H. J. Snaith, Science, 2012, 338, 643-647.

7 H.-S. Kim, I. Mora-Seró, V. González-Pedro, F. FabregatSantiago, E. J. Juarez-Perez, N.-G. Park and J. Bisquert, Nat. Commun., 2013, 4, 2242.

8 A. Yella, H.-W. Lee, H. N. Tsao, C. Yi, A. K. Chandiran, Md. K. Nazeeruddin, E. W.-G. Diau, C.-Y. Yeh, S. M. Zakeeruddin and M. Grätzel, Science, 2011, 334, 629-634.

9 Z. Pan, K. Zhao, J. Wang, Y. Feng and Z. Zhong, ACS Nano, 2013, 7, 5215-5222.

10 J. Wang, I. Mora-Seró, Z. Pan, K. Zhao, H. Zhang, Y. Feng, G. Yang, X. Zhong and J. Bisquert, J. Am. Chem. Soc., 2013, 135, 15913-15922.

11 A. J. Nozik, Physica E, 2002, 14, 115-120.

12 I. Mora-Seró and J. Bisquert, J. Phys. Chem. Lett., 2010, 1, 3046-3052.

13 H. Zhang, K. Cheng, Y. M. Hou, Z. Fang, Z. X. Pan, W. J. Wu, J. L. Hua and X. H. Zhong, Chem. Commun., 2012, 48, 11235-11237.

14 N. Guijarro, J. M. Campiña, Q. Shen, T. Toyoda, T. LanaVillarreal and R. Gómez, Phys. Chem. Chem. Phys., 2011, 13, 12024-12032.

15 M. Shalom, S. Rühle, I. Hod, S. Yahav and A. Zaban, J. Am. Chem. Soc., 2009, 131, 9876-9877.

16 N. Guijarro, Q. Shen, S. Giménez, I. Mora-Seró, J. Bisquert, T. Lana-Villarreal, T. Toyoda and R. Gómez, J. Phys. Chem. C, 2010, 114, 22352-22360. 
17 N. Guijarro, T. Lana-Villarreal, Q. Shen, T. Toyoda and R. Gómez, J. Phys. Chem. C, 2010, 114, 21928-21937.

18 I. Barceló, J. M. Campiña, T. Lana-Villarreal and R. Gómez, Phys. Chem. Chem. Phys., 2012, 14, 5801-5807.

19 S. Giménez, T. Lana-Villarreal, R. Gómez, S. Agouram, V. Muñoz-Sanjosé and I. Mora-Seró, J. Appl. Phys., 2010, 108, 064310.

20 I. Barceló, N. Guijarro, T. Lana-Villarreal and R. Gómez, in Quantum Dot Solar Cells, ed. J. Wu and Z. M. Wang, Springer, New York, 2014, ch. 4, pp. 1-38.

21 Q. Shen, J. Kobayashi, L. J. Diguna and T. Toyoda, J. Appl. Phys., 2008, 103, 084304.

22 I. Barceló, T. Lana-Villarreal and R. Gómez, J. Photochem. Photobiol., A, 2011, 220, 47-53.

23 N. Guijarro, T. Lana-Villarreal, I. Mora-Seró, J. Bisquert and R. Gómez, J. Phys. Chem. C, 2009, 113, 4208-4214.

24 S. Giménez, I. Mora-Seró, L. Macor, N. Guijarro, T. LanaVillarreal, R. Gómez, L. J. Diguna, Q. Shen, T. Toyoda and J. Bisquert, Nanotechnology, 2009, 20, 295204.

25 K. Zídek, K. Zheng, M. Abdellah, N. Lenngrenm, P. Chábera and T. Pullerits, Nano Lett., 2012, 12, 6393-6399.

26 J. B. Sambur, T. Novet and B. A. Parkinson, Science, 2010, 330, 63-66.

27 E. M. Barea, M. Shalom, S. Giménez, I. Hod, I. Mora-Seró, A. Zaban and J. Bisquert, J. Am. Chem. Soc., 2010, 132, 6834-6839.

28 C.-F. Chi, P. Chen, Y.-L. Lee, I.-P. Liu, S.-C. Chou, X.-L. Zang and U. Bach, J. Mater. Chem., 2011, 21, 17534-17540.

29 N. Guijarro, T. Lana-Villarreal, T. Lutz, S. A. Haque and R. Gómez, J. Phys. Chem. Lett., 2012, 3, 3367-3372.

30 G. Hodes, J. Phys. Chem. C, 2008, 112, 17778-17787.

31 I. Hod, V. González-Pedro, Z. Tachan, F. Fabregat-Santiago, I. Mora-Seró, J. Bisquert and A. Zaban, J. Phys. Chem. Lett., 2011, 2, 3032-3035.

32 L. L. Kazmerski, M. S. Ayyagari and G. A. Sangorn, J. Appl. Phys., 1975, 46, 4865-4869.

33 M. Booth, A. P. Brown, S. D. Evans and K. Critchley, Chem. Mater., 2012, 24, 2064-2070.

34 L. L. Kazmerski and G. A. Sanborn, J. Appl. Phys., 1977, 48, 3178-3180.

35 J. Klaer, J. Bruns, R. Henninger, K. Siemer, R. Klenk, K. Ellmer and D. Bräunig, Semicond. Sci. Technol., 1998, 13, 1456-1458.

36 T. Wilhelm, B. Berenguier, M. Aggour, K. Skorupska, M. Kanis, M. Winkelnkemper, J. Klaer, C. Kelch and H.-J. Lewerenz, Thin Solid Films, 2005, 480-481, 24-28.
37 T.-L. Li, Y.-L. Lee and H. Teng, J. Mater. Chem., 2011, 21, 5089-5098.

38 T.-L. Li, Y.-L. Lee and H. Teng, Energy Environ. Sci., 2012, 5, 5315-5324.

39 P. K. Santra, P. V. Nair, K. G. Thomas and P. V. Kamat, J. Phys. Chem. Lett., 2013, 4, 722-729.

40 J. Luo, H. Wei, Q. Huang, X. Hu, H. Zhao, R. Yu, D. Li, Y. Luo and Q. Meng, Chem. Commun., 2013, 49, 3881-3883.

41 J.-Y. Chang, L.-F. Su, C.-H. Li, C.-C. Chang and J.-M. Lin, Chem. Commun., 2012, 48, 4848-4850.

42 C. Chen, G. Ali, S. H. Yoo, J. M. Kum and S. O. Cho, J. Mater. Chem., 2011, 21, 16430-16435.

43 J.-Y. Chang, J.-M. Lin, L.-F. Su and C.-F. Chang, ACS Appl. Mater. Interfaces, 2013, 5, 8740-8752.

44 L. Li, T. J. Daou, I. Texier, T. T. K. Chi, N. Q. Liem and P. Reiss, Chem. Mater., 2009, 21, 2422-2429.

45 X. Tang, W. Cheng, E. S. G. Choo and J. Xue, Chem. Commun., 2011, 47, 5217-5219.

46 K.-T. Kuo, D.-M. Liu, S.-Y. Chen and C.-C. Lin, J. Mater. Chem., 2009, 19, 6780-6788.

47 H. Nakamura, W. Kato, M. Uehara, K. Nose, T. Omata, S. Otsuka-Yao-Matsuo, M. Miyazaki and H. Maeda, Chem. Mater., 2006, 18, 3330-3335.

48 N. Guijarro, T. Lana-Villarreal and R. Gómez, Chem. Commun., 2012, 48, 7681-7683.

49 L. J. Diguna, Q. Shen, J. Kobayashi and T. Toyoda, Appl. Phys. Lett., 2007, 91, 023116.

50 V. González-Pedro, X. Xu, I. Mora-Seró and J. Bisquert, ACS Nano, 2010, 4, 5783-5790.

51 M. Shalom, S. Buhbut, S. Tirosh and A. Zaban, J. Phys. Chem. Lett., 2012, 3, 2436-2441.

52 T. Berger, T. Lana-Villarreal, D. Monllor-Satoca and R. Gómez, J. Phys. Chem. C, 2007, 111, 9936-9942.

53 T. Berger, D. Monllor-Satoca, M. Jankulovska, T. Lana-Villarreal and R. Gómez, ChemPhysChem, 2012, 13, 2824-2875.

54 D. R. Pernik, K. Tvrdy, J. G. Radich and P. V. Kamat, J. Phys. Chem. C, 2011, 115, 13511-13519.

55 I. Mora-Seró, S. Giménez, F. Fabregat-Santiago, R. Gómez, Q. Shen, T. Toyoda and J. Bisquert, Acc. Chem. Res., 2009, 42, 1848-1857.

56 M. Shalom, S. Dor, S. Rühle, L. Grinis and A. Zaban, J. Phys. Chem. C, 2009, 113, 3895-3898.

57 Y. Lian, F. Zhang, D. Pan, H. Li and Y. Lu, J. Mater. Chem., 2012, 22, 8759-8763. 